\title{
El aumento del crimen y la disminución de la participación laboral en el ingreso en México
}

\author{
Increased Crime and Decreased Labour \\ Participation in the Income in Mexico
}

O aumento do crime e a diminuição da participação do trabalho na renda no México

Daniel Velázquez-Orihuela* René Leticia Lozano-Cortés**

Fecha de recepción: 6 de noviembre del 2018

Fecha de aceptación: 24 de mayo del 2019

Cómo citar este artículo/ To reference this article / Comment citer cet article / Para citar este artigo:

Velázquez-Orihuela, D. \& Lozano-Cortés, R. (2019). El aumento del crimen y la disminución de la participación laboral en el ingreso en México. Apuntes del CENES, 38(68), 49-78. https://doi.org/10.19053/01203053.v38. n68.2019.8573

* Doctor en Ciencias Económicas. Profesor-investigador del Área Académica de Economía de la Universidad Autónoma del Estado de Hidalgo, México. daniel_velazquez7607@uaeh.edu.mx. @ https://orcid.org/0000-0002-0953-9905

** Doctora en Ciencias Económicas. Profesora-investigadora del Departamento de Ciencias Económico Administrativas de la Universidad de Quintana Roo, México. uqroo2@yahoo.com.mx @ https://orcid.org/0000-0003-3679-8141 


\section{Resumen}

El crecimiento del crimen y la reducción del ingreso salarial como parte del ingreso nacional son dos fenómenos que han caracterizado a la economía mexicana desde finales de la década de los setenta hasta la actualidad; sin embargo, usualmente, en la literatura económica se estudian las causas que originan estos fenómenos de forma separada. En contraste, en este artículo se propone un modelo de salarios de eficiencia en un contexto de equilibrio restringido por demanda, con la finalidad de mostrar que ambos fenómenos tienen una causa común: la disminución en las oportunidades de los trabajadores de insertarse y permanecer empleados en el mercado laboral.

Palabras clave: distribución del ingreso, crimen, desempleo, salarios, mercado de trabajo.

Clasificación JEL: D33, E19, E25, E24, K42. 


\begin{abstract}
The growth of crime and the decline of wage share are two trends that have characterized the Mexican economy since the late seventies until the present. However, in the economy research, the causes of these trends are studied in a separated way. In contrast, in this paper is proposed a model of efficiency wages in framework of demand-constrained equilibrium, in order to show that the growth in the crime supply and the decrease of wage share have a common cause: the diminution of the opportunities for workers to insert themselves and remain employed in the labor market.
\end{abstract}

Keywords: distribution of income, crime, unemployment, wages, labour market. 


\section{Resumo}

O crescimento do crime e a redução da renda salarial como parte da renda nacional são dois fenômenos que caracterizaram a economia mexicana desde o final dos anos setenta até o presente. No entanto, geralmente, na literatura econômica, as causas que originam esses fenômenos são estudadas separadamente. Em contraste, neste artigo propõe-se um modelo de salários de eficiência num marco de equilíbrio restringido pela demanda, a fim de mostrar que ambos os fenômenos têm uma causa comum: a diminuição nas oportunidades para os trabalhadores se inserirem e permanecerem empregados em o mercado de trabalho.

Palavras-chave: distribuição de renda, crime, desemprego, salários, mercado de trabalho. 


\section{INTRODUCCIÓN}

El crecimiento en el crimen y la reducción de la participación de los salarios en el producto interno bruto (PIB) son dos fenómenos que han caracterizado la economía mexicana en las últimas déca- das. Las tendencias claramente inversas entre el porcentaje de la participación salarial en el ingreso nacional y la tasa de robos por cada cien mil habitantes se pueden observar en la Figura 1.

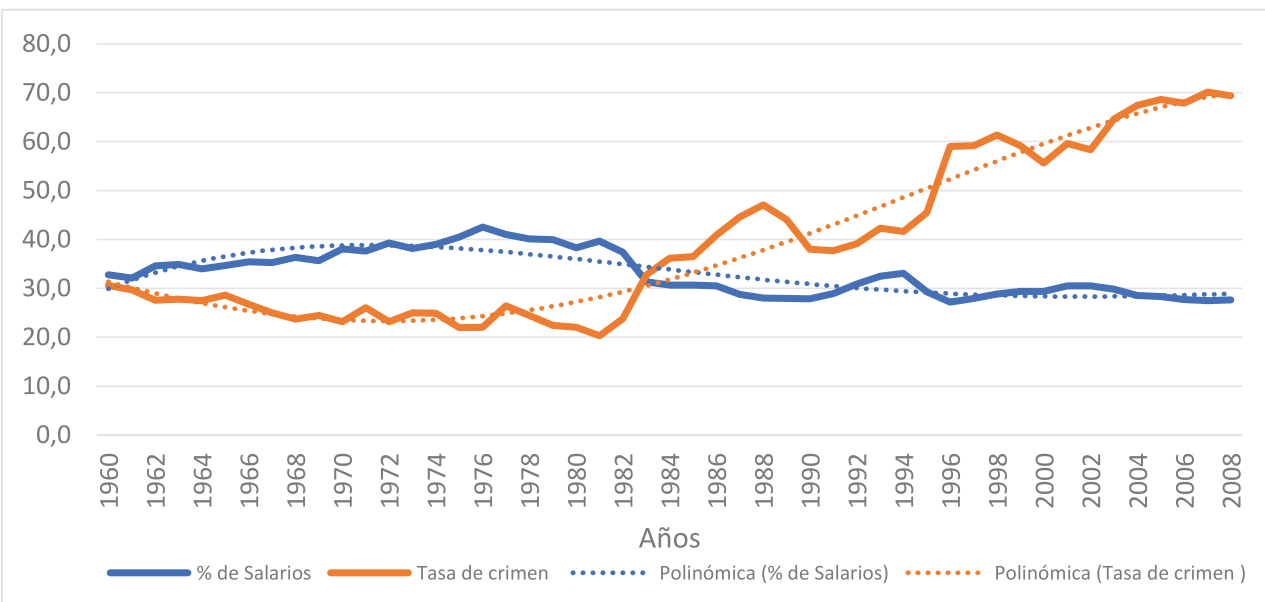

Figura 1. Participación salarial en el PIB y tasa de robos por cada 100000 habitantes en México.

Fuente: elaboración propia con base en Piccato, Hidalgo y Lajous (2018), y Luyando y Herrera (2018).

En esta figura, la línea azul muestra la participación de los salarios como porcentaje del PIB en México. Se observa que esta tuvo una tendencia creciente hasta 1976, después empezó a disminuir, cayendo aceleradamente a principios de los ochenta y mediados de los noventa. Por otra parte, la línea naranja muestra la tasa de robos por cada cien mil habitantes y por los cuales se ha iniciado 
un proceso ante el ministerio público'; se observa que esta sigue una tendencia en contrasentido a la participación salarial, disminuyendo en los períodos que creció la participación salarial y aumentando cuando esta decreció.

Existen dos formas plausibles de explicar las tendencias en contrasentido que se observan entre la evolución de la participación salarial y la tasa de robos:

1. Postular que la caída en la participación salarial provoca el crecimiento en la tasa de robos. Esta idea implica que la distribución asimétrica del ingreso motiva a las personas a delinquir; en la literatura económica, esta explicación ha sido ampliamente explorada (Enamorado \& Winkler, 2016; Kelly, 2006).

2. Argumentar que existe una causa común que origina tanto la caída en la participación salarial como el crecimiento en la tasa de robos. Esta idea ha sido poco estudiada, pues usualmente en la literatura económica se suelen examinar las causas que originan estos fenómenos de forma separada, es decir, como si fuesen ajenos uno del otro.

Por supuesto, ambas explicaciones no son excluyentes. Sin embargo, es metodológicamente conveniente estudiarlas por separado. El presente artículo tiene por objetivo contribuir a la segunda, es decir, ofrecer un argumento que sustente que existe una causa común tanto al crecimiento de la tasa de robos como a la caída en la participación salarial.

Para ello se desarrolla un modelo teórico que ofrece una explicación común al crecimiento de la oferta de crimen y a la reducción de la participación del ingreso salarial en el ingreso nacional. La hipótesis de este artículo es que una reducción en las oportunidades de los trabajadores de ingresar y permanecer empleados en el mercado laboral puede ocasionar que la oferta de crimen aumente y que el ingreso salarial disminuya como proporción del ingreso nacional.

Este artículo se divide en cuatro secciones: la primera es la presente introducción, después se realiza una breve revisión de la literatura del crimen y de la distribución del ingreso, en la tercera sección se construye un modelo de salarios de eficiencia en un escenario de equilibrio restringido por demanda, con la finalidad de mostrar que existen causas comunes al crecimiento de la criminalidad y la concentración del ingreso. En particular se argumenta que si las oportunidades de los trabajadores de ingresar y permanecer ocupados en el mercado laboral se reducen, entonces la oferta de crimen aumentará y la participación del ingreso salarial en el ingreso total disminuirá. En la última sección se ofrecen las conclusiones del artículo.

1 Estos delitos son considerados del fuero común. 


\section{REVISIÓN DE LA LITERATURA}

Desde finales de la década de los setenta y principios de la década de los ochenta se inició una tendencia que marca una caída en la participación de los salarios en el ingreso nacional (IMF, 2007; ILO \& OECD, 2015). Esta tendencia se ha observado tanto en los países industrializados como en los emergentes. Sin embargo, esta ha sido más acelerada en los países emergentes y pobres, que en los países ricos (ILO, 2011; Stockhammer, 2013).

Según el Fondo Monetario Internacional (IMF, por sus siglas en inglés), la reducción de los salarios en el ingreso nacional se debe principalmente al cambio tecnológico (IMF, 2007). Esta idea se sustenta en la teoría neoclásica, la cual postula que los factores se remuneran conforme a su productividad. En consecuencia, los cambios en la productividad del trabajo explican cómo se modifica la participación del ingreso laboral en el $\mathrm{PIB}^{2}$. Esta idea es fuertemente cuestionada por la Organización Internacional del Trabajo (ILO), pues según esta organización, la reducción de la participación del ingreso salarial en el PIB se debe principalmente a la financiarización $y$, en menor medida, a la globalización, a la reducción del Estado de bienestar y a la flexibilización del mercado de trabajo (ILO, 2011; Stockhammer, 2013).
La financiarización es la principal causa que explica el porqué ha caído la participación del ingreso salarial en países emergentes (Stockhammer, 2013). Uno de los principales mecanismos que tiene esta para reducir la participación de los salarios en el PIB son las macrodevaluaciones.

La globalización permite que las empresas puedan moverse libremente de un país a otro en busca de reducir sus costos laborales, por lo que los trabajadores se ven obligados a competir con trabajadores de otros países, lo cual reduce el poder de negociación de los sindicatos y con ello la participación de los trabajadores en el ingreso nacional (Stockhammer, 2013).

La reducción del Estado de bienestar y la flexibilización del mercado de trabajo usualmente se ven como mecanismos que disminuyen el poder de los sindicatos. Por un lado, la reducción del Estado de bienestar suele estar asociado con una menor red de seguridad social tanto para los trabajadores empleados como para los desempleados; por otro lado, la flexibilización del mercado laboral suele implicar reducir los costos de despido y contratación a partir de privilegiar los contratos individuales sobre los colectivos, atenuando así la influencia de los sindicatos en las empresas (ILO, 2011).

2 La idea de que los cambios en la productividad del trabajo explican cómo varia la participación del trabajo en la renta se utiliza en la teoría del crecimiento económico para explicar cómo se distribuye el ingreso entre trabajo calificado y no calificado. Una revisión interesante de esta literatura se encuentra en Kahhat (2011). 
La escuela poskaleckiana retoma la idea clásica y marxista de que la distribución del ingreso se da en una pugna entre clases sociales antagónicas (Hein, 2017). Se resalta la idea de que la distribución del ingreso entre capitalistas y trabajadores se explica fundamentalmente por el poder de los monopolios y de los sindicatos. Siempre que los primeros incrementen su poder, la distribución favorecerá a los capitalistas; pero si son los sindicatos los que incrementan su poder, la distribución favorecerá a los trabajadores.

Por otra parte, en la revisión de la literatura de economía del crimen se encuentra que, usualmente, el análisis de los determinantes de la oferta de crimen parte de asumir que el delincuente es un agente racional que busca maximizar su utilidad y, por tanto, antes de tomar la decisión de realizar un acto delictivo, lleva a cabo una evaluación costo-beneficio (Becker, 1968; Ehrlich, 1973).

Becker (1968) describe la oferta de crimen de un individuo como una función de la probabilidad de ser arrestado, del castigo que recibiría el delincuente si es declarado culpable y de otros factores que influyen en la disposición de los individuos para cometer un delito. Por su parte, Ehrlich (1973) presenta una función de oferta de delitos que, además de incluir la probabilidad de ser aprehendido y castigado, contiene los ingresos de las actividades legales e ilegales, así como la tasa de desempleo y otras variables que pueden afectar la frecuencia de cometer delitos; las cuales pueden ser el nivel de bienestar del delincuente o el de su familia, la eficiencia en su autoprotección, la cantidad de inseguridad para su familia o para su organización criminal, la severidad de las penas, el encarcelamiento o una combinación de ambas.

Yamada, Yamada y Kang (1991) señalan que además de considerar la elección entre dedicarse a actividades legales o ilegales, en el análisis de la oferta de crimen deben tenerse en cuenta las diferencias individuales dadas sus preferencias en cuanto al riesgo y al ocio. Supone que los criminales se caracterizan por su alta preferencia por el riesgo y el ocio. Además, argumenta que un cambio en los salarios de los empleados afecta el desempleo y el número de criminales. En cuanto a la cantidad de crímenes que se estaría dispuesto a cometer por un desempleado, esta dependerá de la utilidad que obtiene por delinquir, la cual es el resultado de la pérdida (en dinero) por ser aprehendido y castigado, más los ingresos que logra por realizar actividades ilegales.

Machin y Megheir (2004), en su estudio, amplían el modelo básico de Ehrlich (1973) al considerar que los individuos no solo hacen una elección óptima entre trabajar en actividades ilegales o legales, comparando los rendimientos por delinquir con respecto a los costos por no trabajar, sino que es necesario considerar también a los individuos que combinan ambas actividades, es decir, 
que al mismo tiempo que trabajan en actividades legales dedican tiempo a realizar actividades ilegales, en otras palabras, las actividades ilegales y legales no son sustitutas perfectas.

Machin y Meghir (2004) y Yamada et al. (1991) incluyen un sistema de transferencias a personas de bajos ingreso o desempleados como un elemento más para sopesar por los individuos que enfrentan un problema de elección respecto al tiempo que deciden asignar a la realización de actividades legitimas o ilegitimas, cuando la actividad criminal y el trabajo legal son actividades sustitutas que compiten por el tiempo disponible del individuo, las transferencias tienen un efecto ingreso y afectan los retornos del trabajo cuando se está fuera del mercado laboral (desempleado). Cuando las transferencias por desempleo son mayores que los salarios que se obtienen por actividades legales, los individuos pueden decidir abandonar su trabajo para ser desempleados de manera voluntaria.

Para Becker (1968) y Ehrlich (1973), la función de oferta de crimen individual tiene las mismas propiedades en el nivel agregado y, por tanto, en un país la probabilidad de que un delincuente sea arrestado y castigado, de ser encontrado culpable, tendrá una relación negativa con el número de delitos, y el efecto de la variable compuesta por las oportunidades dependerá de la variable que se modifique, que pueden ser el desempleo y los salarios.
Altindag (2012) señala que un individuo que considera que en la actualidad o en el futuro tendrá mejores oportunidades en actividades legales, será menos propenso a realizar actividades ilegales. Esas oportunidades se pierden cuando se presenta una recesión económica $y$, por tanto, son altas las tasas de desempleo en el mercado laboral de actividades legales, entonces un individuo puede elegir delinquir.

Por su parte, Yamada (1985) considera que los cambios en las condiciones del mercado de trabajo legal alteran las oportunidades de los beneficios legales que un individuo puede obtener. El desempleo permite que los costos de oportunidad por delinquir sean menores y, por tanto, aumenta la preferencia por cometer un delito. Para Liu, Fullerton y Ashby (2013), los individuos decidirán no realizar actividades ilegales cuando los beneficios derivados del crimen disminuyen o los costos aumentan (se incluyen principalmente las ganancias de actividades legales que no se perciben, así como la probabilidad de ser aprehendido y castigado). Algunas variables del mercado de trabajo afectan los beneficios y los costos esperados del crimen, tales como el ingreso y salarios promedio, mientras que el desempleo afectará principalmente los costos esperados del crimen.

La sociedad mexicana actualmente vive una crisis de inseguridad, la tasa de delitos tanto del fuero federal como 
del común ${ }^{3}$ han experimentado una tendencia al alza desde la década de los ochenta hasta la actualidad (Piccato et al., 2018).

En promedio anual para el periodo 19972018, los delitos denunciados del fuero federal fueron 90.6, mientras que los delitos del fuero común fueron 1425.95, en ambos casos por cada cien mil habitantes. Para este mismo período, los delitos contra la propiedad representan el $54.2 \%$ del total de los delitos del fuero común. Para el 2008, los delitos contra la propiedad crecieron en un 9.78 $\%$, posteriormente descendieron; sin embargo, para el 2017 crecieron en más del $13 \%$. Dentro de los delitos contra la propiedad, el robo representa el $76 \%$ de estos.

Es importante mencionar que tanto los delitos del fuero común como los del fuero federal tienen un comportamiento diferenciado según sea la entidad fede- rativa que integra el territorio nacional. Si bien las cifras sobre el crimen son alarmantes, estos datos subestiman el problema de inseguridad, ya que muchos de los crímenes cometidos no se denuncian debido principalmente a la desconfianza que las víctimas del delito tienen a las autoridades. Según la Encuesta Nacional de Victimización y Percepción de la Seguridad Pública 2017, no se denuncia el $93 \%$ de los delitos cometidos (INEGI, 2017).

Por otra parte, en la última mitad del siglo XX, la financiarización tiene un papel importante para explicar la caída de la participación del ingreso salarial en el ingreso nacional en México. De forma análoga a lo argumentado por Stockhammer (2013), se observa que los períodos de las macrodevaluaciones provocaron fuertes procesos inflacionarios, los cuales redujeron de manera muy importante los salarios reales (véase Tabla 1)

Tabla 1. Evolución del tipo de cambio, precios y salarios reales en las crisis financieras

\begin{tabular}{cccc}
\hline \multirow{2}{*}{ Período } & \multicolumn{3}{c}{ Tasas de crecimiento } \\
\cline { 2 - 4 } & INPC* $^{*}$ & Tipo de cambio & Salario real promedio \\
\hline $1982-1989$ & $4,460.19$ & $10,035.1$ & -46.59 \\
\hline $1994-1996$ & 79.67 & 153.26 & -26.0 \\
\hline
\end{tabular}

Fuente: elaboración propia con base en datos de Banxico y Samaniego (2014).

* Índice nacional de precios al consumidor

3 En México, los delitos suelen clasificarse en general en dos grandes grupos: delitos del fuero federal y delitos del fuero común. Los delitos del fuero federal son aquellos relacionados con la seguridad nacional y se refieren a la delincuencia organizada, el porte de armas de uso exclusivo del ejército, el narcotráfico y el contrabando, entre otros. Los delitos del fuero común se tipifican por los códigos penales de los gobiernos locales y son aquellos que afectan directamente a las personas y sus bienes, tales como el robo, abuso de confianza, secuestro y homicidio, entre otros (Secretariado Ejecutivo del Sistema Nacional de Seguridad Pública, 2018). 
En la crisis económica de los ochenta, el salario promedio cayó en un $46.59 \%$. Esta fuerte reducción de los salarios reales solo fue posible con la cooperación de los sindicatos, los cuales respaldaron las políticas de contención salarial que los gobiernos mexicanos impusieron en este periodo. A finales de la década de los ochenta, el Gobierno, las principales organizaciones campesinas, el sector empresarial y los principales sindicatos firmaron los "pactos económicos" con la finalidad de contener la inflación inercial. Entre estos pactos se destaca el "Pacto de Solidaridad Económica", el cual estuvo vigente de diciembre 1987 a diciembre 1988. En este pacto, los sindicatos se comprometían a no pedir aumento en el salario mínimo, pese a las altas tasas de inflación, lo cual redujo el salario mínimo real en ese período en un $18.4 \%$ y el salario medio real en un $11.8 \%$.

La proliferación de sindicatos blancos en México ha mermado los derechos laborales de los trabajadores (Ramírez, 2011). Actualmente, la Confederación Sindical Internacional ubica a México entre los países en los cuales los derechos laborales no se garantizan (CSI, 2017).

El papel que desempeñaron las crisis financieras durante las décadas de los ochenta y noventa es muy importante para explicar la caída de la participación salarial en el ingreso nacional. Sin embargo, en las últimas dos décadas la economía mexicana ha gozado de cierta estabilidad cambiaria, lo que le permitido contar con bajas tasas de inflación, las cuales no superan los dos dígitos, incluso durante la recesión del 2009. Para el período 2003-2017, la tasa de inflación promedio anual fue de $4.03 \%$. No obstante, para ese mismo periodo, la participación de los salarios en el ingreso nacional se redujo en 5.55 puntos porcentuales del PIB. Por otra parte, la tasa promedio anual de delitos contra propiedad por cada cien mil habitantes para el período 2003-2017 se mantuvo alta, esta fue de 765.2.

Si bien reconocemos los múltiples factores que influyen tanto la caída de la participación del ingreso salarial en el PIB como del crecimiento en los delitos que atentan contra la propiedad, en este artículo nos enfocamos en estudiar la existencia de una causa común a ambos fenómenos; en particular sostenemos que una reducción en las oportunidades de los trabajadores de ingresar y permanecer empleados en el mercado laboral puede fomentar el incremento de los delitos contra la propiedad - concretamente, los robos-y reducir la participación de los salarios en el ingreso nacional.

De manera análoga a la literatura de la economía del crimen, en este artículo se asumen agentes representativos y racionales, pero, a diferencia de esta literatura, el análisis se realiza asumiendo que las empresas pagan salarios de eficiencia, en un contexto de equilibrio restringido por demanda. El principal 
aporte de este artículo, y que lo diferencia tanto de la literatura de la economía del crimen como de la de distribución del ingreso, es su capacidad para ofrecer al mismo tiempo una explicación del incremento del crimen y de la reducción de la participación del ingreso laboral.

El estudio de la oferta de crimen y de la distribución del ingreso parte de asumir que los trabajadores ven reducidas sus oportunidades de ingresar y permanecer ocupados en el mercado laboral. Este modelo se presenta en la siguiente sección.

\section{MODELO DE SALARIOS DE EFI- CIENCIA EN UN CONTEXTO DE EQUILIBRIO RESTRINGIDO POR DEMANDA}

El presente modelo teórico tiene como objetivo mostrar que el crecimiento en la oferta del crimen y la reducción de la participación del ingreso salarial en el ingreso total tienen una causa común: la disminución de las oportunidades de los trabajadores de ingresar y permanecer ocupados en el mercado laboral. Esta se representa con un aumento en la tasa esperada de desempleo. Para cumplir con este objetivo, el modelo parte de los siguientes supuestos:

Se asume que las personas no tienen certeza sobre la ocurrencia de ciertos eventos, por ejemplo, si delinquen serán castigadas por el sistema judicial o no.
Sin embargo, ante esta incertidumbre, basan sus decisiones en la probabilidad subjetiva de ocurrencia de un evento. Esta se define como la expectativa racional de la probabilidad objetiva de un evento, Así, en el presente modelo, la probabilidad subjetiva de ocurrencia del evento $x$ es $E\left(p_{x}\right)$, donde $E$ es el operador de expectativas racionales y $p_{x}$ es la probabilidad objetiva de que ocurra el evento $x$. Usualmente en la literatura se trabaja con probabilidades objetivas, y la probabilidad de ser aprehendido y castigado no es la única incertidumbre a la que se enfrenta un individuo para decidir si delinque (Becker, 1968; Ehrlich, 1973; Yamada et al., 1991; Mauro \& Carmeci, 2007).

En este análisis, a diferencia de los modelos previos, se incluye el supuesto de que todos los individuos que participan en la economía tienen la misma probabilidad de ser víctimas de la delincuencia $(E(\phi))$ independientemente de si son trabajadores o empresarios, de si están empleados, desempleados, si delinquen o no. Además, por simplicidad se asume que el monto de lo robado $(x)$ es independiente de quién es la víctima del delito.

En esta economía conviven consumidores, empresas y Gobierno. Los primeros pueden dividirse en dos tipos: consumidor-trabajador y consumidor-empresario. Se iniciará el análisis con el comportamiento del consumidor-trabajador. 


\section{Consumidor-trabajador}

El consumidor-trabajador es una persona que no posee derechos de propiedad sobre las empresas, por lo que todos sus ingresos provienen de la forma en que emplee su tiempo, ya sea para delinquir $\left(l_{i}\right)$ o trabajar en una actividad legal $\left(l_{o}\right)$. Adviértase que ambas ocupaciones pueden considerarse como trabajo; no obstante, uno es ilegal y el otro no. A diferencia de Becker (1968) y Ehrlich (1973), pero de forma análoga a Machin y Meghir (2004), en este trabajo se asume que el trabajo legal e ilegal no son sustitutos perfectos. Se supone que todos los delitos que comente están orientados a obtener un ingreso, en consecuencia, son delitos que afectan directamente los derechos de propiedad, por lo que se excluyen los delitos de otro tipo, tales como asesinatos por odio, asesinatos culposos, violación o delitos sexuales, entre otros.

El trabajador, como un agente racional, se enfrenta a la disyuntiva de cómo asignar su tiempo para obtener la mayor cantidad de ingresos posibles. Si trabaja recibirá un salario $(w)$, si delinque obtiene un ingreso $\left(w_{i}\right)^{4}$. Sin embargo, reconoce que si delinque existe la probabilidad $(1-E(q))$ de ser aprehendido y castigado, por lo que se le impondrá una sanción, que para fines de este modelo se asume que la sanción es económica y depende del tiempo que empleó para delinquir $\left(f_{i} l_{i}\right)$. Por otra parte, si opta por ofrecer su trabajo a un empleo legal, existe la probabilidad de que no sea contratado, dicha probabilidad está dada por la expectativa de la tasa de desempleo $(E(u))$. Por lo tanto, el trabajador se enfrenta a la incertidumbre de ser aprehendido y castigado si se dedica a delinquir o a la incertidumbre de no ser contratado y quedar desempleado.

La disyuntiva que enfrenta el consumidor-trabajador entre delinquir o no, implica que existen ocho estados del mundo posibles, con distintas probabilidades de que se verifiquen y diferentes ingresos. A continuación se representan estos en la Tabla 2.

Tabla 2. Estados posibles del mundo

\begin{tabular}{ccc}
\hline Estados del mundo & Probabilidad de ocurrencia & Ingreso esperado \\
\hline $\begin{array}{c}\text { Desempleado, impune y } \\
\text { víctima de la delincuencia }\end{array}$ & $E(u) E(q) E(\varnothing)$ & $w_{i} l_{i}-x$ \\
\hline $\begin{array}{c}\text { Desempleado, no impune y } \\
\text { víctima de la delincuencia }\end{array}$ & $E(u)(1-E(q)) E(\varnothing)$ & $w_{i} l_{i}-f_{i} l_{i}-x$ \\
\hline $\begin{array}{c}\text { Empleado, impune y víctima de } \\
\text { la delincuencia }\end{array}$ & $(1-E(u)) E(q) E(\varnothing)$ & $w_{i} l_{i}+w l_{o}-x$ \\
\hline
\end{tabular}

4 En nuestro modelo, igual que Ehrlich (1973), asumimos que para delinquir no se requiere entrenamiento y, por lo tanto, no hay costos para dedicarse a las actividades ilegales. 


\section{Continuación Tabla 2}

\begin{tabular}{ccc}
\hline $\begin{array}{c}\text { Empleado, no impune y víctima } \\
\text { de la delincuencia }\end{array}$ & $(1-E(u))(1-E(q)) E(\varnothing)$ & $w_{i} l_{i}+w l_{o}-f_{i} l_{i}-x$ \\
\hline $\begin{array}{c}\text { Desempleado, impune y no } \\
\text { víctima de la delincuencia }\end{array}$ & $E(u) E(q)(1-E(\varnothing))$ & $w_{i} l_{i}$ \\
\hline $\begin{array}{c}\text { Desempleado, no impune y no } \\
\text { víctima de la delincuencia }\end{array}$ & $E(u)(1-E(q))(1-E(\varnothing))$ & $w_{i} l_{i}-f_{i} l_{i}$ \\
\hline $\begin{array}{c}\text { Empleado, impune y no víctima } \\
\text { de la delincuencia }\end{array}$ & $(1-E(u)) E(q)(1-E(\varnothing))$ & $w_{i} l_{i}+w l_{o}$ \\
\hline $\begin{array}{c}\text { Empleado, no impune y no } \\
\text { víctima de la delincuencia }\end{array}$ & $(1-E(u))(1-E(q))(1-E(\varnothing))$ & $w_{i} l_{i}+w l_{o}-f_{i} l_{i}$ \\
\hline
\end{tabular}

Fuente: elaboración propia

A partir de los distintos estados de la naturaleza posibles, se obtiene la utilidad esperada del consumidor: $E U\left(c_{s} l_{s}\right)=$ $\sum_{s=a}^{n} E\left(p_{s}\right) U\left(c_{s}\right)$. Donde $c_{s}$ es el consumo que se obtiene en el estado del mundo $s, E\left(p_{s}\right)$ es la probabilid ad subjetiva de que ese estado ocurra. A diferencia de Ehrlich (1973) y Yamada et al. (1991), el ocio no aparece en la función de utilidad. Esto se debe a que el consumidor-trabajador tiene que ocuparse en actividades legales o ilegales, o en ambas, para poder vivir, cualquiera sea la remuneración de estas, en consecuencia, el ocio es una proporción constante del tiempo biológicamente disponible para trabajar.
Lo anterior implica que la suma del tiempo dedicado al trabajo legal más el ilegal es una proporción constante de su dotación de tiempo $(\Gamma)$, una vez que se ha descontado de esta el tiempo consumido en ocio, es decir, $l_{o}+l_{i}=\Gamma$, donde $\Gamma$ es constante y exógeno. Además, se asume que el consumidor-trabajador es adverso al riesgo, por lo cual, $U\left(c_{s}\right)$ es estrictamente cóncava.

Con fundamento en los estados del mundo descritos, la conducta racional del consumidor-trabajador se representa a partir del siguiente ejercicio de maximización:

$$
\begin{aligned}
& M a ́ x E(u)=E(u) E(q) E(\varnothing) \ln \left(w_{i} l_{i}-x\right)+E(u)(1-E(q)) E(\varnothing) \\
& \ln \left(w_{i} l_{i}-f_{i} l_{i}-x\right)+(1-E(u)) E(q) E(\varnothing) \ln \left(w_{i} l_{i}+w l_{o}-x\right)+ \\
& (1-E(u))(1-E(q)) E(\varnothing) \ln \left(w_{i} l_{i}+w l_{o}-f_{i} l_{i}-x\right)+E(u) E(q) \\
& (1-E(\varnothing)) \ln \left(w_{i} l_{i}\right)+E(u)(1-E(q))(1-E(\varnothing)) \ln \left(w_{i} l_{i}-f_{i} l_{i}\right)+ \\
& (1-E(u)) E(q)(1-E(\varnothing)) \ln \left(w_{i} l_{i}+w l_{o}\right)+(1-E(u))(1-E(q)) \\
& (1-E(\varnothing)) \ln \left(w_{i} l_{i}+w l_{o}-f_{i} l_{i}\right)+\lambda\left(\Gamma-l_{i}-l_{0}\right)
\end{aligned}
$$


Siempre que las soluciones sean interiores, es decir, que $l_{o}>$ y $l_{i}>0$, se obtiene que el consumidor maximiza cuando:

$$
\begin{gathered}
\frac{E(u) E(q) E(\varnothing) w_{i}}{w_{i} l_{i}-x}+\frac{E(u)(1-E(q)) E(\varnothing)\left(w_{i}-f_{i}\right)}{\left(w_{i}-f_{i}\right) l_{i}-x}+\frac{E(u)(1-\emptyset)}{l_{i}}= \\
\frac{(1-E(u)) E(q) E(\varnothing)\left(w-w_{i}\right)}{w l_{o}+w_{i} l_{i}-x}+\frac{(1-E(u))(1-E(q)) E(\varnothing)\left(w-w_{i}+f_{i}\right)}{w l_{o}+\left(w_{i}-f_{i}\right) l_{i}-x}+ \\
\frac{(1-E(u)) E(q)(1-E(\varnothing))\left(w-w_{i}\right)}{w_{i} l_{i}+w l_{o}}+ \\
\frac{(1-E(u))(1-E(q))(1-E(\varnothing))\left(w-w_{i}+f_{i}\right)}{\left(w_{i}-f_{i}\right) l_{i}+w l_{o}}
\end{gathered}
$$

$$
\Gamma-l_{i}-l_{0}=0
$$

La ecuación [2] muestra que el consumidor maximiza su función objetivo, cuando la utilidad marginal que obtiene por delinquir es igual a la conseguida por trabajar. La ecuación [3] simplemente muestra que el tiempo disponible una vez descontado el tiempo dedicado al ocio, debe gastarse entre delinquir y trabajar en actividades legales.

\section{Consumidor empresario}

El consumidor-empresario es una persona que posee los derechos de propiedad de las empresas y cuya principal fuente de ingreso no depende del trabajo, sino de las ganancias de las empresas, por lo cual se asume que no oferta trabajo. Los empresarios poseen los recursos y los contactos suficientes para acceder a delitos más rentables y de menor riesgo que los trabajadores, como por ejemplo delitos de cuello blanco, fraudes, corrupción política, entre otros. Sin embargo, este tipo de delitos no se analizará en este documento (forman parte de la agenda de investigación), por lo que se supone que los empresarios no enfrentan la disyuntiva entre delinquir o no.

El ingreso del consumidor empresario depende únicamente de las ganancias de las empresas debido a que no trabaja ni delinque. Él considera las ganancias como dadas, es decir, no dependen de sus decisiones de consumo u ocio. En consecuencia, su consumo $\left(c^{e}\right)$ equivale a la ganancia que recibe de las empresas, (П) menos el costo que paga si fue víctima de algún delito. Lo anterior implica que el consumo agregado de los empresarios equivale a la ganancia de todas las empresas menos el egreso de aquellos empresarios que fueron víctimas de algún delito, es decir: $c^{e}=\Pi-\mathrm{E}(\phi) \mathrm{x}$. 


\section{Empresa representativa}

Se asumirá que la empresa representativa solo produce un bien $\left(y_{o}\right)$ y demanda un insumo: el trabajo $\left(l_{d}\right)$. La conducta racional de la empresa se representa a partir del siguiente ejercicio de maximización:

$$
\text { Máx }(1+\pi)=\frac{y_{o}}{w l_{d}}
$$

$$
\text { S. } a \quad y_{o}=A e(w, E(u)) f\left(l_{d}-l^{*}\right)
$$

Donde $f\left(l_{d}-l^{*}\right)$ es una función homogénea de grado $\mu$, donde $0<\mu<1$. $e_{w}^{\prime}>0, e_{w}^{\prime \prime}<0, e_{E(u)}^{\prime}>0, e_{w}^{\prime \prime}<0, e_{w, E(u)}^{\prime \prime}$ $=0$ ye $e(w, 0)>0$.

De las expresiones [4] y [5] se resaltan las siguientes características:

1. La empresa maximiza su tasa de ganancias, es decir, el cociente del valor de sus ventas entre el valor de sus costos. Este postulado contrasta con la hipótesis neoclásica de que las empresas maximizan su ganancia, la cual se define como la diferencia entre el valor de sus ventas y el de sus costos 5 . No obstante, es coherente con el postulado clásico-marxista de que la tasa de ganancia guía las decisiones de las empresas, y con las teorías de evaluación de proyectos de inversión, las cuales destacan las tasas de rentabilidad de la inversión como mecanismos útiles para discriminar entre varios proyectos de inversión.

2. De forma análoga a Shapiro $\mathrm{y}$ Stiglitz (1984) y a Solow (1979), se asume que el esfuerzo de los trabajadores depende positivamente del salario real y de la tasa esperada de desempleo. La razón de esto es que los trabajadores bien remunerados tienden a esforzarse más, pues suelen valorar su empleo; por otra parte, si el desempleo es alto, los trabajadores evitarán holgazanear para no ser despedidos, debido a que es costoso encontrar un nuevo empleo.

3. Las empresas tienen costos de organización $\left(l^{*}\right)$. Estos se definen como el trabajo que emplean las empresas para organizar la producción y que no está asociado a producto positivo, pero que es indispensable para producir. En consecuencia, $y_{o}>0$ si y solo si $l_{d}>l^{*}$. Para la empresa competitiva los costos de organización son un dato. Sin embargo, estos se resuelven en el equilibrio general. De acuerdo con Noriega (2001), los costos de organización dependen del tamaño de mercado; la idea es que entre más ventas

5 En Noriega (2001) se realiza un comparativo de una empresa maximizadora de la ganancia con otra maximizadora de la tasa de ganancia; se encuentra que siempre que las empresas puedan dividir su producción en tantas unidades productivas como requieran y compartan el mismo vector de precios y demanda de insumos, entonces la empresa maximizadora de la tasa de ganancia obtendrá un mayor monto de ganancia que la otra empresa. 
tenga la empresa, más organización requiere para atender los distintos contratos.

Partiendo de las ecuaciones [4] y [5] resulta que la empresa maximiza cuando:

$$
\begin{gathered}
\frac{A e(w, u) f\left(l_{d}-l^{*}\right)}{l_{d}}=f_{l_{d}}^{\prime} \\
\frac{e_{w}^{\prime}}{e(w, u)} w=1
\end{gathered}
$$

La ecuación [6] muestra que el productor maximiza su tasa de ganancia cuando el producto medio iguala a la productividad marginal del trabajo. Este resultado contrasta con el propio de la tradición neoclásica y keynesiana, que postula que la productividad marginal del trabajo iguala al salario real. No obstante, es habitual en los modelos que postulan empresas maximizadoras de la tasa de ganancia (Velázquez \& Hernández, 2018; Vargas, 2017; Noriega, 2001).

La ecuación [7] es la ecuación de Solow y muestra que las empresas obtienen las máximas ganancias cuando la elasticidad salario-esfuerzo es igual a la unidad. Al salario que satisface esa identidad se le llama de eficiencia. Esta condición de maximización implica que las empresas ofrecen un salario independientemente de la oferta y demanda de trabajo.
A partir del teorema de Euler sobre funciones homogéneas ${ }^{6}$ se sabe que $\mu y_{o}=f^{\prime}\left(l_{d}-l^{*}\right)$, sustituyendo esta expresión en la ecuación [6], se arriba a:

$$
l_{d}=\left(\frac{1}{1-\mu}\right) l^{*}
$$

La ecuación [8] es la demanda de trabajo, la cual no depende del salario real, en otras palabras, es inelástica con respecto al salario real. Este resultado es claramente diferente al que se obtiene habitualmente en la teoría neoclásica, según la cual la demanda de trabajo depende negativamente del salario real, es decir, las empresas aumentan su demanda de trabajo a medida que el salario real se reduce. No obstante, es un resultado habitual de los modelos que postulan la maximización de la tasa de ganancia, por lo que es análogo al obtenido por Velázquez y Hernández (2018), Noriega (1997) y Noriega (2001).

Sustituyendo la ecuación [8] en [5] se obtiene la oferta de producto: $y_{o}=A e$ $(w, E(u)) f\left(\frac{\mu}{1-\mu} l^{*}\right)$. Esta, de manera similar a la demanda de trabajo, no depende del salario real y está determinada por los costos de organización. Por lo que un análisis más profundo de los planes de compra y venta del productor implica conocer cómo se determinan los costos de organización, lo cual se realizará en el equilibrio general.

6 El teorema de Euler para funciones homogéneas argumenta que si $f\left(x_{1}, x_{2}, x_{3}, \ldots, x_{n}\right)$ es una función homogénea de grado $\lambda$, entonces $\lambda f\left(x_{1}, x_{2}, x_{3}, \ldots, x_{n}\right)=\sum_{i=1}^{n} \frac{d f}{d x_{i}} x_{i}$ 


\section{Gobierno}

Se asume que las sanciones económicas que el sistema judicial impone como castigo a las personas que atrapa delinquiendo $\left((1-E(q)) f_{i} l_{i}\right)$ es el único ingreso del Gobierno. Suponiendo que el Gobierno gasta en consumo $(g)$, pero no otorga transferencias a desempleados y no incurre en déficit, se obtiene: $g=(1-E(q)) f_{i} l_{i}$.

\section{Demanda agregada}

El consumo agregado de los trabajadores $\left(c^{t}\right)$ es la suma del consumo en todos los estados del mundo posible ponderado por su probabilidad de que este se verifique. Así $c^{t}=\sum_{s=1}^{8} E\left(p_{s} c_{s}\right.$. Debido a que el trabajador utiliza todos sus ingresos disponibles para financiar su consumo, se obtiene: $c^{t}=(1-E(u))$ $w l_{o}+w_{i} l_{i}-.(1-E(q)) f_{i} l_{i}-\phi x . \mathrm{El}$ consumo agregado de los trabajadores depende del ingreso que obtuvieron tanto por actividades legales como ilegales, menos los pagos por sanciones económicas de aquellos que fueron aprehendidos y castigados por el sistema judicial por cometer actividades ilícitas, y las pérdidas de aquellos que fueron víctimas del delito.

La demanda agregada $\left(y_{d}\right)$ está integrada por la suma del consumo de los trabajadores, más el consumo de los empresarios, más el gasto del Gobierno, es decir, $y_{d}=c^{t}+c^{e}+\mathrm{g}$.
Para el agregado, las actividades ilícitas no representan un ingreso adicional. La razón de esto es que las ganancias de los delincuentes son pérdidas para las víctimas del crimen, es decir, $w_{i} l_{i}=2 E(\phi)$. De manera análoga, el castigo económico que se les impone a los delincuentes que son atrapados por el sistema judicial es un egreso para ellos y un ingreso para el Gobierno. Así, el crimen es un juego de suma cero, en donde las ganancias de uno son pérdidas para el otro.

A partir del párrafo anterior se tiene que la demanda agregada es: $y_{d}=$ $(1-E(u)) w l_{o}+\Pi$. Es decir, la demanda agregada equivale a la remuneración que las personas esperan obtener por actividades legales, en otras palabras, al ingreso que los trabajadores esperan obtener por emplearse en las empresas y a las ganancias que las empresas otorgan a las personas que son dueñas de los derechos de propiedad de estas. Lo anterior tiene dos importantes implicaciones:

a. El producto de las actividades ilícitas no aparece en la demanda agregada. La razón de esto es que, en el nivel agregado, el crimen es una actividad redistributiva del ingreso, pero no generadora de riqueza, ya que lo que son ganancias para unos, son pérdidas para otros.

b. La demanda agregada depende de la tasa esperada de desempleo. Adviértase que a medida que las expectativas 
se verifiquen, la demanda agregada convergerá con la demanda efectiva $\left(\hat{y}_{d}\right)$. Esta última se define como el plan de demanda que los consumidores y el gobierno pueden financiar, es decir, la demanda efectiva está determinada por el ingreso que las personas realmente obtuvieron. Por lo que solo cuando $E(u)=u$ ambas demandas son iguales.

\section{Equilibrio restringido por demanda}

En la literatura usualmente se indica que los equilibrios restringidos por demanda surgen debido a que los precios se ajustan lentamente, por lo que las personas efectúan transacciones a precios que no son de equilibrio (Argandoña, Gámez \& Mochón, 1995). A diferencia de esta literatura, pero de forma análoga a Velázquez y Hernández (2018), Velázquez y Rodríguez (2016), el equilibrio restringido por demanda surge debido a que los salarios son fijados por la conducta optimizadora de las empresas independientemente de la oferta y demanda de trabajo (ecuación 7), y a que la demanda de trabajo de las empresas no depende del salario real (ecuación 8). Así, no hay un mecanismo de mercado que garantice el equilibrio de pleno empleo. Ante ello, las empresas simplemente ajustan su producción a la demanda vigente.

A partir de este momento se asume que el salario es el de eficiencia, es decir, el que garantiza la máxima ganancia a las empresas. El equilibrio restringido por demanda se verifica a medida que las empresas ajustan su producción a la demanda, es decir: $y_{d}-y_{o}=0$. Sustituyendo la demanda agregada en la condición de equilibrio en el mercado de bienes se obtiene: $(1-E(u)) w l_{o}+$ $\Pi-y_{o}=0$. Adviértase que por definición $\Pi=y_{o}-w l_{d}$, sustituyendo esta definición en la ecuación anterior se obtiene:

$$
(1-E(u)) l_{o}-l_{d}=0
$$

De la ecuación [9] es importante resaltar los siguientes aspectos:

a. En escenarios de desempleo o pleno empleo, la demanda de trabajo equivale a la oferta de trabajo empleada, es decir, $(1-u) l_{o}=l_{d}$. Por lo que el equilibrio restringido por demanda se verifica siempre que los agentes acierten en sus expectativas, y es independiente del monto de los salarios y del valor de la tasa de desempleo.

b. Siempre que la expectativa de la tasa de desempleo se verifique, la demanda agregada será igual a la demanda efectiva. Por lo que el equilibrio restringido por demanda se garantiza cuando los productores ajustan su producción a la demanda efectiva vigente, es decir: $\hat{y}_{d}=y_{o}$.

La razón por la cual el equilibrio restringido por demanda no depende del monto de salarios se debe a que este 
último se fija independientemente de la oferta y demanda de trabajo (ecuación [7]) y a que la demanda de trabajo no depende del salario real (ecuación [8]). Por lo que no hay mecanismos de mercado que garanticen el equilibrio de pleno empleo.

Partiendo de un equilibrio restringido por demanda, se procede a analizar cómo se determina el nivel de empleo. Para ello es necesario conocer cómo se fijan los costos de organización. Sustituyendo la ecuación de oferta de producto en la condición de equilibrio restringido por demanda $\left(\hat{y}_{d}=y_{o}\right)$ se obtienen los costos de organización: $l^{*}=$ $\frac{(1-\mu)}{\mu} j\left(\frac{\hat{y}_{d}}{A e(w, E(u))}\right)$. Donde $j=f^{-1}$, Por lo que $j$ es una función homogénea de grado $\frac{1}{\mu}$. En consecuencia, los costos de organización tienen una relación positiva con la demanda efectiva. Noriega (2001) argumenta que la razón de esto es porque a medida que las empresas se enfrentan a un mercado en expansión, requieren aumentar la organización para hacer frente a una mayor cantidad de contratos y transacciones que se generan por el crecimiento de la demanda. Sustituyendo los costos de organización en la demanda de trabajo se obtiene:

$$
l_{d}=\frac{1}{\mu} j\left(\frac{\hat{y}_{d}}{A e(w, E(u))}\right)
$$

La ecuación (10) es la demanda de trabajo y tiene características muy simila- res a la función ocupación de Keynes; al igual que esta última, tiene una relación positiva con la demanda efectiva, es decir, a medida que se incrementa la demanda efectiva, las empresas contratan más trabajo. Lo anterior se debe a que las empresas ajustan su producción a la demanda efectiva; así que, cuando esta aumenta, contratan más trabajo para ajustar su producción al alza.

\section{Análisis de la distribución y de la oferta de crimen}

Con la finalidad de analizar cómo se modifica la oferta del crimen y la distribución funcional del ingreso, se supondrá que las personas esperan que se reduzcan sus oportunidades de ingresar y permanecer en el mercado laboral, es decir, se asumirá que los trabajadores esperan que la tasa de desempleo aumente. Para hacer viable este análisis se asumirá que la demanda de las familias es financiada con el ingreso que obtuvieron en el período próximo pasado. La idea que sustenta esta aseveración es que los consumidores trabajan durante un período (semana, quincena), al final de este reciben su salario, posteriormente con este ingreso financian su consumo del siguiente período. Para hacer operativa esta idea se plantea que los ingresos de las personas dependen de las actividades económicas legales o ilegales que realizaron en el período 
próximo pasado, aunque es importante recordar que los ingresos de unos (delincuentes) son pérdidas de otros (víctimas), el ingreso de los delincuentes no modifica el ingreso agregado $y$, por tanto, no está presente en la ecuación de la demanda efectiva.

El párrafo anterior implica que la demanda efectiva está determinada por el nivel de empleo y la ganancia de las empresas vigentes en el período pasado, los cuales se consideran conocidos y fijos. Esta idea se formaliza en la siguiente ecuación:

$$
\hat{y}_{d}=\left(1-u_{-1}\right) l_{o-1} w+\Pi_{-1}
$$

En la ecuación [11] el subíndice -1 indica que la variable se determinó en el período pasado.

\section{Reducción en las oportunidades de inserción y permanencia en el merca- do laboral y distribución del ingreso}

Como ya se anotó, la reducción en las oportunidades de ingreso y permanencia de los trabajadores en el mercado laboral se modelan a partir de asumir que los agentes esperan que la tasa de desempleo aumente. La expectativa de una mayor tasa de desempleo obliga a los trabajadores a esforzarse más, debido a que se incrementa la probabilidad de ser despedidos si son sorprendidos holgazaneando. El incremento en el esfuerzo de los trabajadores permite que las empresas reduzcan los salarios. La razón de esto es que ya no es necesario motivar a los trabajadores con salarios altos para que se esfuercen, pues ellos han incrementado su esfuerzo para no ser despedidos. A partir de la ecuación [7] se muestra cómo se reducen los salarios ante un incremento de la tasa esperada de desempleo: $\frac{d w}{d E(u)}=\frac{e_{E(u)}^{\prime}}{e_{w}^{\prime \prime} w}<0$.

La reducción de los salarios contrae la demanda efectiva debido a que disminuye el poder de compra de los trabajadores, con base en [11] se obtiene ese resultado: $\frac{d \hat{y}_{d}}{d E(u)}=\left(1-u_{-1}\right) l_{o-1} w_{E(u)}^{\prime}<0$.

La producción disminuye debido a que las empresas producen solo aquello que el mercado les demanda, a partir de la igualdad demanda efectiva-producción $\left(\hat{y}_{d}=y_{o}\right)$. Se obtiene: $\frac{d y_{o}}{d E(u)}=$ $\left(1-u_{-1}\right) l_{o-1} w_{E(u)}^{\prime}<0$.

La reducción en la demanda efectiva y el cambio en el esfuerzo de los trabajadores afectan la demanda de trabajo. Con la finalidad de analizar cómo cambia el empleo se diferencia la ecuación [10] con respecto a la demanda de trabajo y a la expectativa de la tasa de desempleo, obteniendo:

$$
\begin{gathered}
\frac{d l_{d}}{d E(u)}=\frac{1}{\mu} j^{\prime} \\
{\left[\frac{\hat{y}_{d, E(u)}^{\prime}}{A e(w, E(u))}-\frac{\hat{y}_{d}\left(e_{w}^{\prime} w_{E(u)}^{\prime}+e_{E(u)}^{\prime}\right)}{A e(w, E(U))}\right]<0}
\end{gathered}
$$

El primer término de la ecuación [12] muestra que el empleo disminuye debi- 
do a que las empresas contratan menos trabajo para ajustar su producción a la menor demanda efectiva. Este razonamiento es análogo al que resulta de la función de ocupación de Keynes. No obstante, a diferencia de esta última, la reducción en el nivel de empleo, que en rendimientos decrecientes implica un incremento en la productividad del trabajo, no implica un cambio en los salarios, pues estos están determinados por la ecuación de Solow.

El segundo término indica cómo el cambio en el esfuerzo modificó la demanda de trabajo. Hay dos fuerzas contrarias que alteran el esfuerzo de los trabajadores: por un lado, el incremento en la tasa de desempleo motiva a los trabajadores a esforzase más, por otro, la caída en los salarios desincentiva a los trabajadores a realizar un mayor esfuerzo. No es posible saber a priori qué fuerza dominará. Sin embargo, sí es posible determinar características generales de la función esfuerzo para conocer que fuerza dominará. A partir de la ecuación de Solow se obtiene que si $\left|e_{w, w}^{\prime \prime} w\right| \gtreqless e_{w}^{\prime}$ entonces $\left[e_{w}^{\prime} w^{\prime}+e_{E(u)}^{\prime}\right] \lesseqgtr 0$. En este artículo se asumirá que $\left|e_{w, w}^{\prime \prime} w\right|>e_{w}^{\prime}$ por lo cual $\left[e_{w}^{\prime} w^{\prime}+e_{E(u)}^{\prime}\right]>0$. Es decir, los trabajadores se esfuerzan más ante la expectativa de una mayor tasa de desempleo, pese a la reducción en el salario real. Esta característica de la función esfuerzo es coherente con la evidencia estadística para México, la cual muestra que los trabajadores mexicanos son de los que más horas trabajan (más se esfuerzan) en la OCDE, pese a los bajos salarios recibidos. El mayor esfuerzo de los trabajadores reduce la demanda de trabajo, puesto que se requiere menos trabajo para obtener la misma cantidad de producto.

Adviértase que la reducción en el nivel de empleo y en los salarios reales implica que la masa salarial se redujo. Sin embargo, a priori, esto no implica que los trabajadores hayan reducido su participación en el ingreso total, porque la producción también disminuyó.

Para analizar cómo cambió la participación de los trabajadores en el ingreso total, se partirá de la definición de masa salarial por unidad de producto: $\frac{\mathrm{w} l_{d}}{y_{o}}$. Sustituyendo la ecuación [8] en [5], para expresar la oferta de producto en términos de demanda de trabajo se obtiene: $y_{o}=e(w, E(u)) A f\left(\mu l_{d}\right), \quad$ empleando esta expresión se obtiene:

$$
\frac{w l_{d}}{y_{o}}=\frac{w g\left(l_{d}, \mu\right)}{A e(w, E(u))}
$$

En la expresión [13], $\frac{l_{d}}{f\left(\mu l_{d}\right)}=g\left(l_{d}, \mu\right)$ por lo que $g\left(l_{d}, \mu\right)$ es una función homogénea de grado $1-\mu$ para el dominio de $l_{d}$. Diferenciando [13] respecto a la expectativa de desempleo se obtiene:

$$
\begin{gathered}
\frac{d \frac{w l_{d}}{y_{o}}}{d E(u)}=\left[\frac{w_{E(u)}^{(-)}}{A e(w, E(u))}-\frac{w\left(e_{w}^{(+)} w_{E(u)}^{(-)}+e_{E(u)}^{(+)}\right)}{A e(w, E(u))^{2}}\right] \\
g(w, E(u))+\frac{w}{A e(w, E(u))} g_{l_{d}}^{(+)} l_{d, E(u)}^{(-)}
\end{gathered}
$$


En la ecuación [14], los supraíndices $(+)$ y (-) se utilizan para indicar el signo de la derivada. Sustituyendo la ecuación [7] en [14] se obtiene:

$$
\begin{gathered}
\frac{d \frac{w l_{d}}{y_{o}}}{d E(u)}=-\left[\frac{w e_{E(u)}^{(+)}}{A e(w, E(u))^{2}}\right] g(w, E(u))+ \\
\frac{w}{A e(w, E(u))} g_{l_{d}^{(+)}} l_{d, E(u)}^{(-)}<0
\end{gathered}
$$

La expresión [15] muestra que la participación de los salarios en el ingreso total disminuyó, pese a la reducción en la producción. Esto se debe a dos razones: 1) la caída en los salarios y en el empleo, es decir, la reducción en el ingreso de los trabajadores; 2) el incremento en el esfuerzo y los rendimientos decrecientes a escala de la función de producción que implican que la productividad del trabajo aumentó, aunque que los salarios disminuyeron, por lo que los frutos de la mayor productividad fueron apropiados por las empresas.

Reducción en las oportunidades de inserción y permanencia en el mercado laboral y la oferta de crimen

La reducción en las oportunidades de empleo y en la remuneración de las actividades legales que realizan los trabajadores modifica la oferta de crimen, puesto que para que un individuo decida participar en actividades ilegales hace un análisis costo-beneficio. El deterioro en los salarios conlleva una reducción de las oportunidades en el mercado laboral $\mathrm{y}$, por lo tanto, altera los incentivos para que los individuos decidan participar más en actividades criminales y menos en actividades legales.

Con la finalidad de analizar cómo cambia la oferta de crimen se sustituye la ecuación [5] en [4], para expresar la condición de equilibrio del consumidor en términos de oferta de crimen. Adviértase que la oferta de crimen está en función de la tasa esperada de desempleo y del salario real, pero este último también está determinado por la tasa esperada de desempleo, es decir, $l_{i}=j(E(u), w(E(u)))$ por lo que:

$\frac{d l_{i}}{d E(u)}=\frac{d j(E(u)}{d E(u)}+\frac{d j(w(E(u))}{d w} \frac{d w}{d E(u)}$

Para facilitar el análisis, primero se estudiará cómo cambia la oferta de crimen cuando se incrementa la tasa esperada de desempleo y todo lo demás (incluido salario) permanece constante; después se procederá a analizar cómo varía la oferta de crimen cuando se incrementa el salario y todo lo demás permanece constante. Siempre que se considere que los salarios se redujeron a consecuencia de un incremento en la tasa esperada de desempleo, la suma de las dos diferenciales equivaldrá a la ecuación [16]. 


$$
\begin{gathered}
\frac{d l_{i}}{d E(u)}=\left[\frac{E(q) E(\emptyset)\left(w-w_{i}\right)}{w_{i} l_{i}+w\left(\Gamma-l_{i}\right)-x}+\frac{(1-E(q)) E(\emptyset)\left(w-w_{i}+f_{i}\right)}{\left(w_{i}-f_{i}\right) l_{i}+w\left(\Gamma-l_{i}\right)-x}+\frac{E(q)(1-E(\varnothing))\left(w-w_{i}\right)}{w_{i} l_{i}+w\left(\Gamma-l_{i}\right)}+\right. \\
\left.\frac{(1-E(q))(1-E(\emptyset))\left(w-w_{i}+f_{i}\right)}{\left(w_{i}-f_{i}\right) l_{i}+w\left(\Gamma-l_{i}\right)}+\frac{E(q) E(\emptyset) w_{i}}{w_{i} l_{i}-x}+\frac{(1-E(q)) E(\varnothing)\left(w_{i}-f_{i}\right)}{\left(w_{i}-f_{i}\right) l_{i}-x}+\frac{(1-E(\phi))}{l_{i}}\right] \div \\
{\left[\frac{(1-E(u)) E(q) E(\phi)\left(w-w_{i}\right)^{2}}{\left(w_{i} l_{i}+w\left(\Gamma-l_{i}\right)-x\right)^{2}}+\frac{(1-E(u))(1-E(q)) E(\varnothing)\left(w-w_{i}-f_{i}\right)^{2}}{\left(\left(w_{i}-f_{i}\right) l_{i}+w\left(\Gamma-l_{i}\right)-x\right)^{2}}+\frac{(1-E(u)) E(q)(1-E(\varnothing))\left(w-w_{i}\right)^{2}}{\left(w_{i} l_{i}+w\left(\Gamma-l_{i}\right)\right)^{2}}+\right.} \\
\left.\frac{(1-E(u))(1-E(q))(1-E(\varnothing))\left(w-w_{i}+f_{i}\right)^{2}}{\left(\left(w_{i}-f_{i}\right) l_{i}+w\left(\Gamma-l_{i}\right)\right)^{2}}+\frac{E(u) E(q) E(\varnothing) w_{i}^{2}}{\left(w_{i} l_{i}-x\right)^{2}}+\frac{E(u)(1-E(q)) E(\varnothing)\left(w_{i}-f_{i}\right)^{2}}{\left(\left(w_{i}-f_{i}\right) l_{i}-x\right)^{2}}+\frac{(1-E(\phi))}{l_{i}^{2}}\right]
\end{gathered}
$$

La ecuación [17] muestra cómo cambia la oferta del crimen cuando las personas esperan que la tasa de desempleo aumente y todo lo demás permanece constante. El signo del divisor de la expresión [17] es estrictamente positivo; por lo que el signo de la ecuación depende del dividendo. Adviértase que los diferentes denominadores de los términos que integran el dividendo son los ingresos que obtienen el consumidor-trabajador en los distintos estados de la naturaleza, y se asumirá que estos son positivos. En consecuencia, el signo de la ecuación [17] depende del numerador de los diferentes términos que integran el dividendo, en estos se focaliza el análisis. El estudio se divide en dos partes, en la primera se estudia cómo responden a la oferta de crimen de los consumidores que se encuentran empleados, en la segunda, de los consumidores que están desempleados.

1. Los numeradores de los primeros cuatro términos del dividendo $\left(E(q) E(\varnothing) \quad\left(w-w_{i}\right), \quad(1-E(q))\right.$ $E(\varnothing)\left(w-w_{i}+f_{i}\right), E(q)(1-E(\varnothing))$ $\left(w-w_{i}\right)$ y $(1-E(q))(1-E(\varnothing))$ $\left.\left(w-w_{i}+f_{i}\right)\right)$ determinan cómo cambia la oferta de crimen cuando se incrementa la tasa esperada de desempleo y los consumidores están empleados en los distintos estados de la naturaleza. El signo de estos depende del tamaño relativo del salario, es decir, siempre que el salario sea mayor (menor) (igual) que los ingresos netos de las actividades delictivas, en los distintos estados de la naturaleza, entonces un incremento en la tasa esperada de desempleo aumentará (reducirá) (no modificará) la oferta de crimen de los consumidores empleados. Para entender esto se propone el siguiente ejemplo: supongamos que los consumidores esperan que la tasa de desempleo se reduzca, es decir, que haya más empleo, entonces ellos reducirán su oferta de crimen si el ingreso que obtienen por las actividades legales es mayor al de las actividades ilegales, para los distintos estados de la naturaleza. La razón de esto es que reducir su oferta de crimen implica aumentar la oferta de 
trabajo, lo cual solo harán si obtienen un mayor ingreso por esta actividad.

2. El numerador de los últimos tres términos del dividendo $\left(\left(E(q) E(\varnothing) w_{i},\right),(1-E(q)) E(\varnothing)\right.$ $\left(w_{i}-f_{i}\right)$ y $\left.(1-E(\phi))\right)$ indica cómo reaccionan los consumidores desempleados ante un incremento en la tasa esperada de desempleo; su reacción no depende del salario, está determinada por el ingreso que se obtiene de las actividades ilícitas.

3. Para analizar cómo cambia la oferta de crimen a consecuencia de un incremento en el salario, se procede de forma análoga al análisis anterior, es decir, se sustituye [5] en [4] y se diferenciará la ecuación, tal que:

$$
\begin{aligned}
\frac{d l_{i}}{d w} & =-\left[\frac{\left(1-E(u) E(q) E(\varnothing)\left(w_{i} \Gamma-x\right)\right.}{\left(w_{i} l_{i}+w\left(\Gamma-l_{i}\right)-x\right)^{2}}+\frac{(1-E(u))(1-E(q)) E(\phi))\left(\Gamma\left(w_{i}-f_{i}\right)-x\right)}{\left(\left(w_{i}-f_{i}\right) l_{i}+w\left(\Gamma-l_{i}\right)-x\right)^{2}}\right. \\
+ & \left.\frac{(1-E(u)) E(q)(1-E(\phi)) w_{i} \Gamma}{\left(w_{i} l_{i}+w\left(\Gamma-l_{i}\right)\right)^{2}}+\frac{(1-E(u))(1-E(q))(1-E(\phi)) \Gamma\left(w_{i}-f_{i}\right)}{\left(\left(w_{i}-f_{i}\right) l_{i}+w\left(\Gamma-l_{i}\right)\right)^{2}}\right] \\
& \div\left[\frac{(1-E(u)) E(q) E(\phi)\left(w-w_{i}\right)^{2}}{\left(w_{i} l_{i}+w\left(\Gamma-l_{i}\right)-x\right)^{2}}+\frac{(1-E(u))(1-E(q)) E(\varnothing)\left(w-w_{i}-f_{i}\right)^{2}}{\left(\left(w_{i}-f_{i}\right) l_{i}+w\left(\Gamma-l_{i}\right)-x\right)^{2}}\right. \\
+ & \frac{(1-E(u)) E(q)(1-E(\varnothing))\left(w-w_{i}\right)^{2}}{\left(w_{i} l_{i}+w\left(\Gamma-l_{i}\right)\right)^{2}}+\frac{(1-E(u))(1-E(q))(1-E(\varnothing))\left(w-w_{i}+f_{i}\right)^{2}}{\left(\left(w_{i}-f_{i}\right) l_{i}+w\left(\Gamma-l_{i}\right)\right)^{2}} \\
& \left.+\frac{E(u) E(q) E(\varnothing) w_{i}^{2}}{\left(w_{i} l_{i}-x\right)^{2}}+\frac{E(u)(1-E(q)) E(\varnothing)\left(w_{i}-f_{i}\right)^{2}}{\left(\left(w_{i}-f_{i}\right) l_{i}-x\right)^{2}}+\frac{(1-E(\phi))}{l_{i}^{2}}\right]<0
\end{aligned}
$$

Siempre que el ingreso neto de las actividades ilícitas $\left(w_{i} l_{i}\right.$ para cuando el delincuente queda impune y $w_{i} l_{i}-f_{i} l_{i}$ para cuando no hay impunidad) sea positivo, un incremento en el salario reducirá la oferta de crimen.

Las ecuaciones [17] y [18] muestran que, ante un incremento en la tasa esperada de desempleo, aumenta la oferta de crimen, porque se reducen las oportunidades de trabajo legal y la remuneración de este. Este resultado es similar al de Liu Yu et al. (2013), quienes señalan que algunas variables del mercado de trabajo afectan los beneficios y los costos esperados del crimen, tales como el ingreso y salarios promedio, mientras que el desempleo afectará principalmente los costos esperados del crimen.

\section{CONCLUSIONES}

En este artículo se propuso un modelo de salarios de eficiencia en contexto de equilibrio restringido por demanda. En este marco analítico se muestra que la reducción de la participación de los sa- 
larios en el ingreso total y el incremento de la oferta de crimen pueden tener una causa común: la disminución de las oportunidades de los trabajadores de acceder y permanecer ocupados en el mercado laboral. Esta última se modela a partir de asumir que los agentes esperan que la tasa de desempleo aumente.

El mecanismo a través del cual un aumento en la tasa esperada de desempleo reduce la participación de los salarios en el ingreso total es el siguiente: cuando los trabajadores esperan que el desempleo aumente, se esfuerzan más por temor a perder su trabajo si son sorprendidos holgazaneando; dado el mayor esfuerzo que realizan los trabajadores, las empresas ya no tienen incentivos para pagar salarios altos, pues los trabajadores ya se están esforzando, por lo que reducen el salario. La disminución del salario contrae el poder de compra de los trabajadores y, con ello, la demanda efectiva; las empresas entonces ajustan su producción a la baja para hacerla coincidir con la menor demanda efectiva, para lo cual contratan menos trabajo y, por con- siguiente, generan desempleo. La caída en los salarios y en el empleo reduce el ingreso de los trabajadores y los costos de las empresas, en contraste, el mayor esfuerzo que realizan los trabajadores y los rendimientos decrecientes a escala de la función de producción aumenta la productividad del trabajo y, con ello, se incrementa la ganancia de las empresas. Es así como los salarios reducen su participación en el ingreso, mientras que las ganancias aumentan la suya.

Por otro lado, el incremento en la tasa esperada de desempleo y la reducción de los salarios, que esta provocó, modifican los costos y beneficios que evalúan las personas antes de tomar la decisión sobre delinquir o no. Se encuentra que una disminución de los salarios tiene un efecto positivo en la oferta de crimen, debido a que disminuyen los beneficios legales frente a los ilegales; mientras que la tasa de desempleo actúa sobre los costos de delinquir, en el sentido de que una pérdida en las oportunidades en el mercado laboral disminuye los costos de oportunidad de las actividades ilegales. 


\section{REFERENCIAS}

Altindag, D. (2012). Crime and Unemployment: Evidence from Europe. International Review of Law and Economics, 32(1), 145-157. https://doi.org/10.1016/j. irle.2011.10.003

Argandoña, A., Gámez, C. \& Mochón, F. (1995). Macroeconomía avanzada I. Modelos dinámicos y teoría de la política económica. Madrid: McGraw-Hill.

Becker, G.S. (1968). Crime and Punishment: An Economic Approach. Journal of Political Economy, 76(2), 1-55. Retrieved from https://olis.leg.state.or.us/ liz/2017R1/Downloads/CommitteeMeetingDocument/125036

CSI. (2017) Índice global de los derechos de la CSI 2017. Los peores países del mundo para los trabajadores y las trabajadoras. Bruselas: Confederación Sindical Internacional. Recuperado de https://www.ituc-csi.org/ indice-global-de-los-derechos-2017?lang=en

Ehrlich, I. (1973). Participation in Illegitimate Activities: Theoretical and Empirical Investigation. Journal of Political Economy, 81(3), 145-157. Retrieved from https://www.journals.uchicago.edu/doi/10.1086/260058

Enamorado, T. \& Winkler, H. (2016). Income Inequality and Violent Crime: Evidence from Mexico's Drug War. Journal of Development Economics, 120, 128-143. https://doi.org/10.1016/j.jdeveco.2015.12.004

Hein, E. (2017). Post-Keynesian Macroeconomics since the Mid-1990s- Main Development. European Journal of Economics and Economic Police: Intervention, 14(2), 131-172. https://doi.org/10.4337/ejeep.2017.02.01

ILO (2011). Making Markets Work for Jobs. World of Work Report 2011. Retrieved from https:/www.ilo.org/wcmsp5/groups/public/@dgreports/@dcomm/@ publ/documents/publication/wcms_166021.pdf

ILO \& OECD (2015). The Labour Share in G20 Economies. In Report Prepared for the G20 Employment Working Group Antalya, Turkey, 26-27 february 2015. Retrieved from https://www.oecd.org/g20/topics/employment-and-social-policy/The-Labour-Share-in-G20-Economies.pdf 
IMF. (2007). The Globalization of Labor. In IMF (Ed.). World Economic Outlook: Spillovers and Cycles in the Global Economy. Washington D. C.: IMF. Retrieved from file://C:/Users/84861\%20HP_ODM002/AppData/Local/Packages/Microsoft.MicrosoftEdge_8wekyb3d8bbwe/TempState/Downloads/_textpdf\%20(1). pdf

INEGI. (2017). Encuesta nacional de victimización y percepción sobre seguridad pública (ENVIPE) 2017. México: INEGI. Recuperado de https://www.inegi. org.mx/programas/envipe/2017/

Kahhat, J. (2011). La desigualdad de los ingresos laborales. La demanda y oferta de competencias. En L. López-Calva \& N. Lusting (Ed.) La disminución de la desigualdad en la América Latina (pp. 43-62). México: Fondo de Cultura Económica.

Kelly, M. (2006). Inequality and Crime. Review of Economics and Statistics, 82(4), 530-539. https://doi.org/10.1162/003465300559028

Liu, Y., Fullerton, T., M. \& Ashby, J. (2013). Assessing the Impacts of Labor Market and Deterrence Variables on Crime Rates in México. Contemporary Economic Policy, 31(4), 669-690. https://oi.org/10.1111/j.1465-7287.2012.00339.x

Luyando, C. \& Herrera J. (2018). Distribución funcional del ingreso y régimen de crecimiento en México y España. Cuadernos de Economía, 37(73), 121-142. https://doi.org/10.15446/cuad.econ.v37n73.58811

Machin, S. \& Meghir, C. (2004). Crime and Economic Incentives. The Journal of Human Resource, 39(4), 958-979. https://doi.org/10.3368/jhr.XXXIX.4.958

Mauro, L. \& Carmeci, G. (2007). A Poverty Trap of Crime and Unemployment. Review of Development Economics, 11(3), 450-462. https://doi. $\operatorname{org} / 10.1111 / \mathrm{j} .1467-9361.2006 .00350 . \mathrm{x}$

Noriega, F. A. (1997). Teoría del desempleo y la distribución. Evidencia empírica: México 1984-1994. Investigación Económica, 57(22), 143-185. Recuperado de https://www.jstor.org/stable/42842382?seq=1\#page_scan_tab_contents

Noriega, F. A. (2001). Macroeconomía para el desarrollo: teoría de la inexistencia del mercado de trabajo. México: McGraw-Hill. 
Piccato, P., Hidalgo, S. \& Lajous, A. (2018). Estadísticas del crimen en México: series históricas 1926-2008. Recuperado de https://ppiccato.shinyapps.io/judiciales/

Ramírez, M. (2011) Los sindicatos blancos en Monterrey. Frontera Norte, 23(46), 177-210. http://dx.doi.org/10.17428/rfn.v23i46.828

Samaniego, N. (2014) La participación del trabajo en el ingreso nacional: el regreso a un tema olvidado. Economía Unam, 11(33) 52-77. Recuperado de https://www. sciencedirect.com/science/article/pii/S1665952X14721814

Shapiro, C. \& Stiglitz, J. E. (1984). Equilibrium Unemployment as a Worker Discipline Device. The American Economic Review, 74(3), 433-444. Retrieved from http://kisi.deu.edu.tr/yesim.kustepeli/shapiro1984.pdf

Solow, R. M. (1979). Another Possible Source of Wage Stickiness. Journal of Macroeconomics, 1(1), 79-82. https://doi.org/10.1016/0164-0704(79)90022-3

Stockhammer, E. (2013). Why Have Wage Shares Fallen? A Panel Analysis of Determinants of Functional Income Distribution. OIL Condition of Work and Employment Series, 35, 1-52. Retrieved from https://www.ilo.org/public/libdoc/ ilo/2013/470913.pdf

Vargas, J. R. (2017). Trabajo especializado y diferencial salarial. Análisis Económico, 32(80), 27-44. Recuperado de https://www.redalyc.org/html/413/41352782003/

Velázquez, D. \& Hernández, Z. S. (2018). Una explicación a la curva de salarios. Cuadernos de Economía, 37(73), 67-94. https://doi.org/10.15446/cuad.econ. v37n73.54225

Velázquez, D. \& Rodríguez, E. (2016). Una explicación a la relación procíclica y anticíclica de los salarios ante el empleo. Equilibrio Económico, 12(2), 125160. Recuperado de http://www.equilibrioeconomico.uadec.mx/descargas/ Rev2016/Rev16Sem2Art1.pdf

Yamada, T. (1985). The Crime Rate and the Condition of the Labor Market: A Vector Autoregressive Model. NBER Working Paper, (1782), 1-18. Retrieved from https://www.nber.org/papers/w1782.pdf 
Yamada, T., Yamada, T. \& Kang, M. (1991). Crime Rates versus Labor Market Conditions: Theory and Time-Series Evidence. NBER Working Paper, (3801), 1-26. Retrieved from https://www.researchgate.net/profile/Tetsuji_Yamada/ publication/5191776_Crime_Rates_Versus_Labor_Market_Conditions_Theory_and_Time-Series_Evidence/links/0912f5091cde9c6cf1000000.pdf 


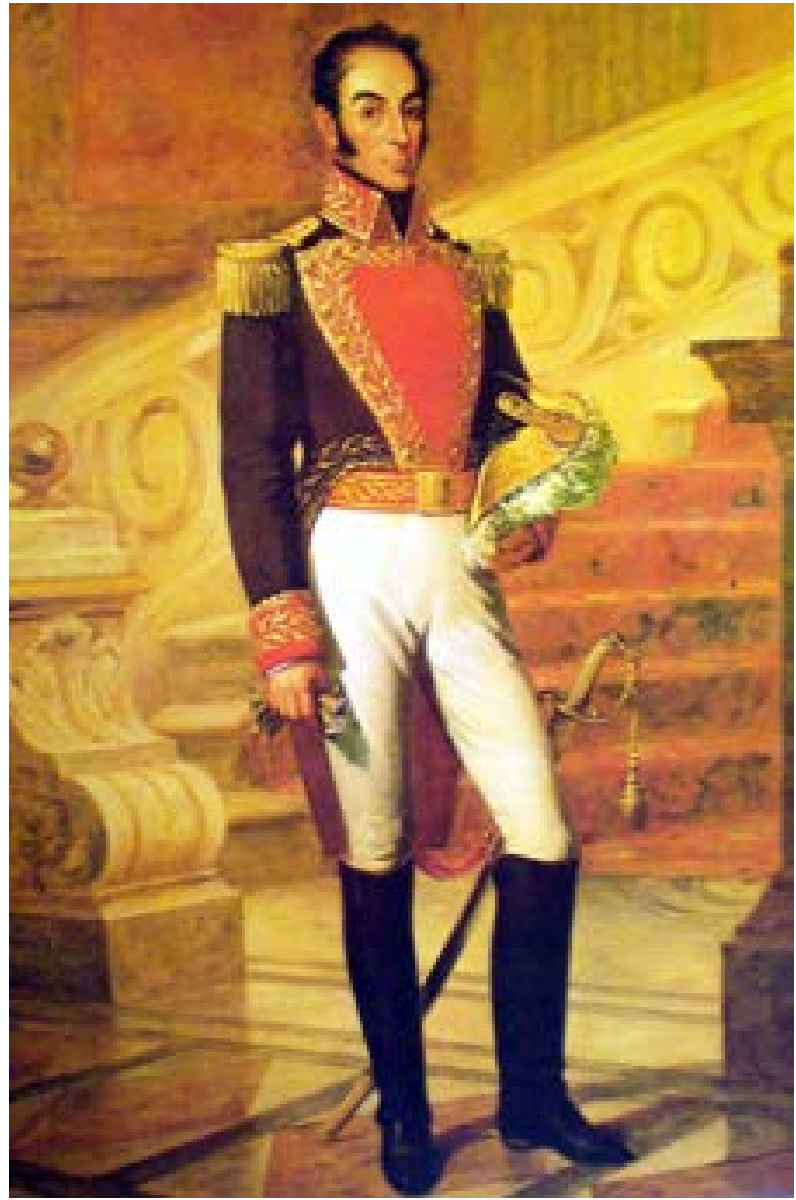

General Simón Bolívar

POLÍTICA ECONÓMICA 
